\title{
The importance of intra-hospital pharmacovigilance in the detection of medication errors
}

\author{
Francisco Villegas, ${ }^{1}$ David Figueroa-Montero, ${ }^{2}$ Varenka Barbero-Becerra, ${ }^{2}$ Eva Juárez-Hernández, ${ }^{2}$ \\ Misael Uribe, ${ }^{2}$ Norberto Chávez-Tapia ${ }^{2}$ and Octavio González-Chon ${ }^{3}$ \\ 'Department of Pharmacovigilance; ${ }^{2}$ Translational Research Unit; ${ }^{3}$ Medical Directorate, Fundación Clínica Médica Sur, Ciudad de México, Mexico
}

\begin{abstract}
Introduction: Hospitalized patients are susceptible to medication errors, which represent between the fourth and the sixth cause of death. The department of intra-hospital pharmacovigilance intervenes in the entire process of medication with the purpose to prevent, repair and assess damages. Objective: To analyze medication errors reported by Mexican Fundación Clínica Médica Sur pharmacovigilance system and their impact on patients. Method: Prospective study carried out from 2012 to 2015, where medication prescriptions given to patients were recorded. Owing to heterogeneity, data were described as absolute numbers in a logarithmic scale. Results: 292932 prescriptions of 56368 patients were analyzed, and $8.9 \%$ of medication errors were identified. The treating physician was responsible of $83.32 \%$ of medication errors, residents of $6.71 \%$ and interns of $0.09 \%$. No error caused permanent damage or death. Conclusion: This is the pharmacovigilance study with the largest sample size reported.
\end{abstract}

KEY WORDS: Pharmacovigilance. Drug monitoring. Medication error. Drug safety.

\section{Introduction}

Over the years, ever since the disaster caused by thalidomide in 1961, pharmacovigilance has gained importance, has been intimately assimilated into medical practice and has become an essential part of public health. Pharmacovigilance is based on scientific principles and is continuously being updated with the emergence of each drug, in order to consider the benefits or risks it might entail for patients. ${ }^{1-3}$

Health professionals, by means of the use of medical prescriptions, seek to attain a beneficial therapeutic outcome for the patient within an ethical, scientific and legal framework. Physicians, based on their knowledge and according to the pathology and needs of patients, prescribe drugs by means of which they hope patients recover both their health and well-being. ${ }^{4}$

Now, not all the responsibility for treatment success or failure falls on the physician, but it is a duty that is shared with other members of health personnel: nurses, pharmacists, pharmacovigilance personnel and trainee physicians. It is also duty of the patient and his/her family knowing the pathology, the prescribed treatment and the risks it entails, ${ }^{5}$ in order to decrease medication error rates and the resulting therapeutic failure.

Hospitalized patients are highly susceptible to medication errors. ${ }^{6}$ Medication error is defined as any incident that can harm the patient, from hospitalization time prolongation to the worst outcome: death.,8 Evidence indicates that medication errors represent between the fourth and the sixth cause of death ${ }^{9}$, and can be attributed to different factors, some of them already identified: verbal instructions,${ }^{10}$ inadequate transcription, ${ }^{8,11,12}$ ignorance of drug-drug interactions, inadequate preparation of medication, missed administration, inappropriate or unreadable medical instructions, inadequate or wrongly-calculated dose, , ,12,13 incorrect dilution, wrong patient identification, incorrect administration route, deficient
Correspondence:

Octavio Gónzalez-Chon

E-mail: ogchon@medicasur.org.mx
Date of reception: 07-04-2016

Date of acceptance: 30-04-2016

DOI://dx.doi.org/10.24875/GMM.M18000126
Gac Med Mex. 2018;154:129-135

Contents available at PubMed www.gacetamedicademexico.com 
Table 1. Error type category and definition

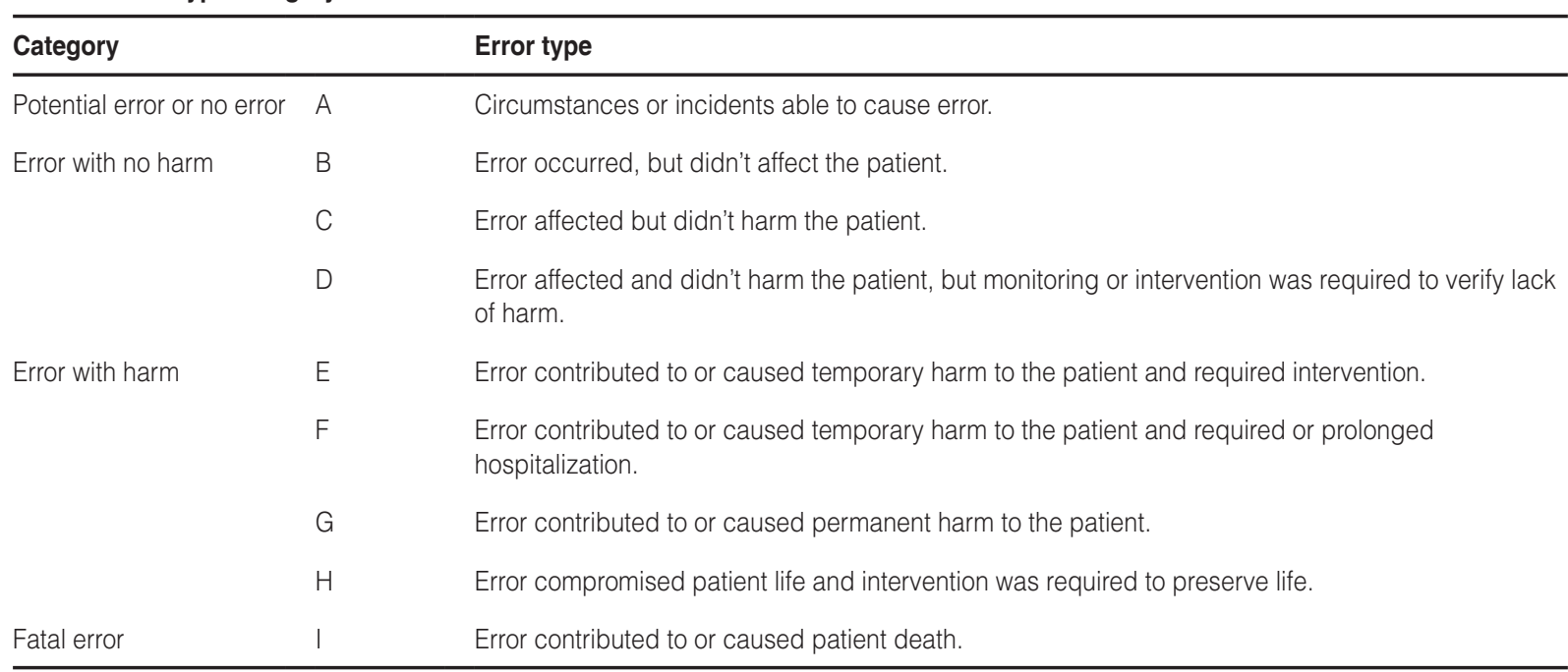

storage of the substance, ${ }^{12}$ lack of pharmacological surveillance, and even increased workload. ${ }^{8}$

In order to decrease medication errors, it is necessary for their causes to be identified and analyzed, for adequate information to be provided to medical and nursing personnel, as well as for support of a pharmacovigilance department to be offered. ${ }^{3}$ Pharmacovigilance personnel, when detecting an error or being informed of any by another member involved in the patient's care, takes care of stopping and correcting the medication process and assessing potential damages or those caused to the patient, in addition to notifying the responsible party about such error.

At Fundación Clínica Médica Sur, the Pharmacology Department has the mission to intervene in the entire medication process in all different areas of the hospital complex. Pharmacovigilance personnel members are in close communication with the personnel that is in contact with patients and their medications, and they even have the mission to be present in the medication process, corroborating that it is adequately carried out and that the patient doesn't experience adverse side effects.

Pharmacovigilance actions, together with health personnel better training, translate into better medical practice, better patient care, medical care costs decrease, shortening of hospital length of stay and continuous updating of all personnel that has contact with patients and their treatment. ${ }^{14}$

The purpose of this investigation was to describe the results of the hospital pharmacovigilance system in the detection of medication errors and their clinical impact on Fundación Clínica Médica Sur patients.

\section{Method}

Study carried out at Fundación Clínica Médica Sur from 2012 to 2015. Data were prospectively captured by members of the Pharmacovigilance Department at each one of the hospital departments ${ }^{15}$ and were classified by the type of consequence caused by the error (Table 1). Information was obtained from patients attended to at different hospital areas: pediatrics, obstetrics and gynecology; 5 floors comprising the hospitalization, intensive therapy, coronary therapy and intermediate therapy areas. Also the departments of emergency, oncology center and hospital areas with reduced number of patients, which were grouped in the "other" section, were analyzed. Necessary medical care and information was provided to patients and their families in order to promote continuous mutual cooperation, with the purpose to detect possible adverse effects and future consequences.

The results are described in absolute numbers on a logarithmic scale owing to numerical heterogeneity. The study was approved by the hospital ethics committee and adhered to the Declaration of Helsinki (1989) guidelines, respecting patient personal information confidentiality.

\section{Results}

In the medication errors analysis per department, data from 56,368 patients were reviewed, 292,932 prescriptions were analyzed and 26,071 medication errors were detected, which corresponded to $8.9 \%$ of total prescriptions. 

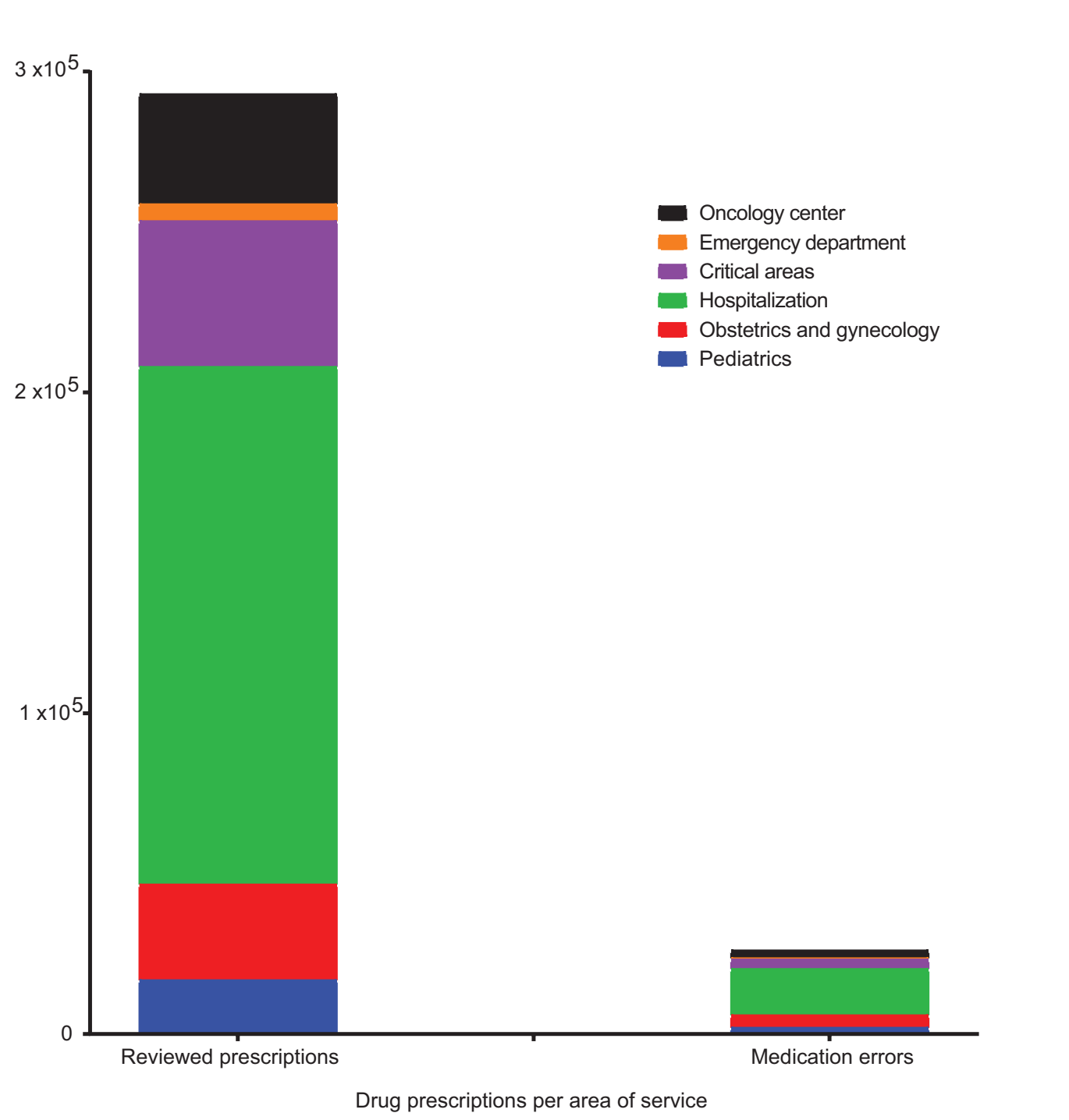

Figure 1. Medication errors and reviewed prescriptions per area of service. Each hospital area is independently represented within the same bar, and is directly proportional to the number of medication errors or reviewed prescriptions.

The area where more prescriptions were reviewed was hospitalization, with 161,319 , and the area with less prescriptions was the emergency department, with 5262. The detected medication errors were directly proportional to the number of prescriptions: in the hospitalization area there were 14,438 errors in comparison with 402 in the emergency department (Figure 1).

During hospital stay, patients are under the care of different members of the department; from the treating physician, who decides the treatment, to the staff physician in charge of the department, nurses, pharmacists, trainee physicians (interns and residents) and other personnel that intervenes in the medication process. Therefore, all those who intervene in drug prescription and administration were named responsible personnel. Figure 2 shows that the treating physician was responsible for $83.32 \%$ of medication errors. Taking the department into account, most errors attributed to doctors occurred in hospitalization areas, and the least occurred in the emergency department. In contrast, resident physicians were responsible for $6.71 \%$ of errors. Finally, the responsible for the least number of errors was the intern physician, with $0.09 \%$ (Figure 2).

With regard to the phases that comprise the process of drug administration, numerous errors originated at the prescription phase, mainly in the hospitalization area. The second most common error occurred during the transcription phase. The third cause of error was detected to be wrong administration: wrong administration route, different pharmaceutical presentation or wrong administration timing (Figure 3). 


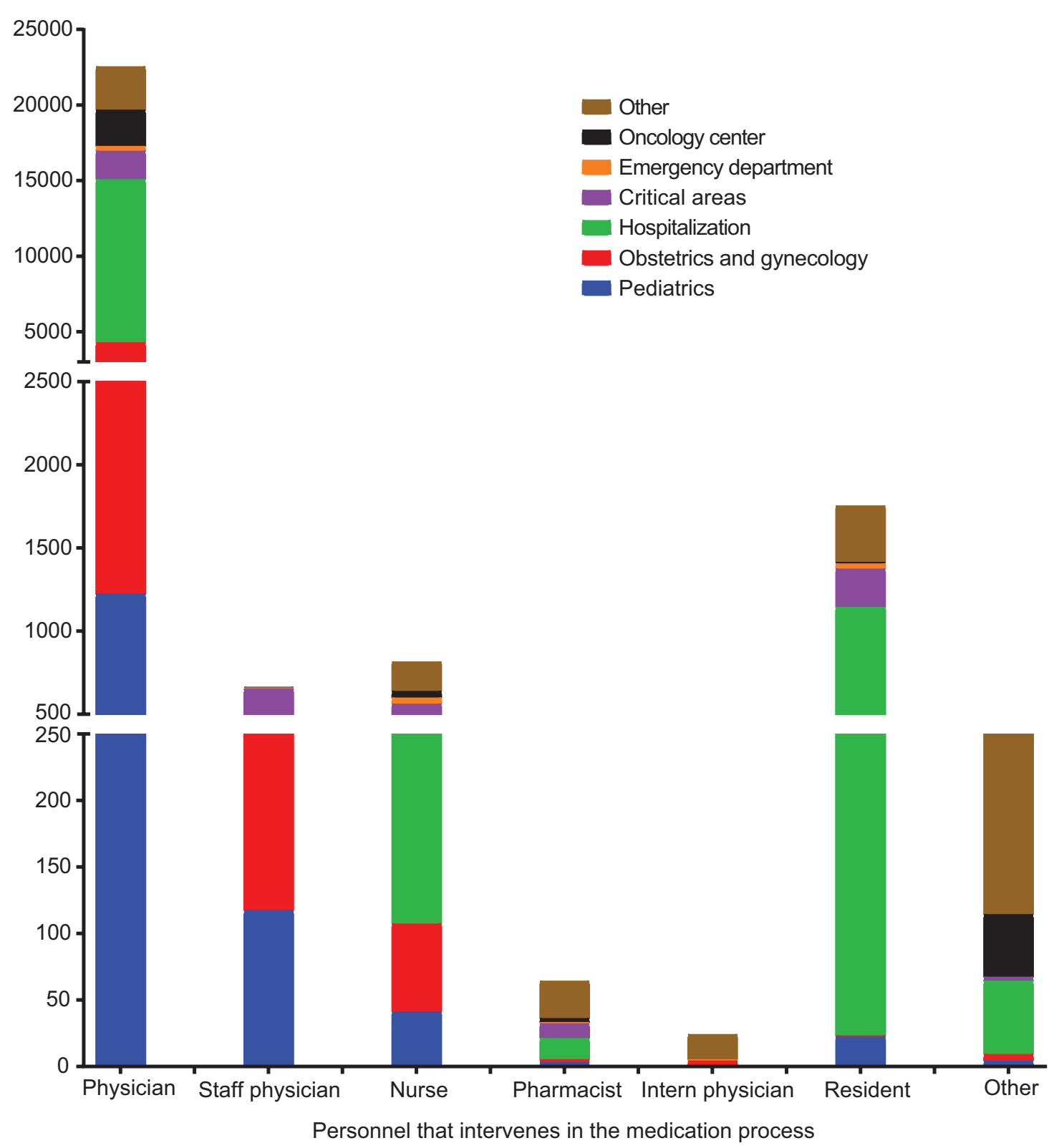

Figure 2. Medication errors by responsible personnel. Each hospital area is independently represented within the same bar and is directly proportional to the number of medication errors according to the responsible personnel member.

Another analyzed aspect were error consequences (Table 1). Consequence $A$ was found to account for $6.04 \%$; $B$, for $29.63 \%, C$, for $54.42 \%, D$, for $9.63 \%$; $E$, for $0.22 \%$ and $F$, for $0.03 \%$. Thus, the most commonly detected consequence was $C$, which represents an error without harm and that doesn't require any intervention even when it affected the patient. Type $B$ consequence, which represents an error that doesn't affect the patient, was second place. Errors that affect the patient comprise type $C$ to $F$ consequences, where outcomes include monitoring, intervention or hospitalization prolongation, with the latter being the worst outcome. No one got to cause permanent damage and no deaths occurred as a result of medication errors (Figure 4).

Interventions carried out by the Pharmacovigilance Department were also assessed and analyzed by area, as well as those that were accepted and rejected. There were more accepted than rejected interventions. More interventions were accepted than rejected; the department where more interventions were carried out was the hospitalization area, whereas the emergency department was where there were the least (Fig. 5). 


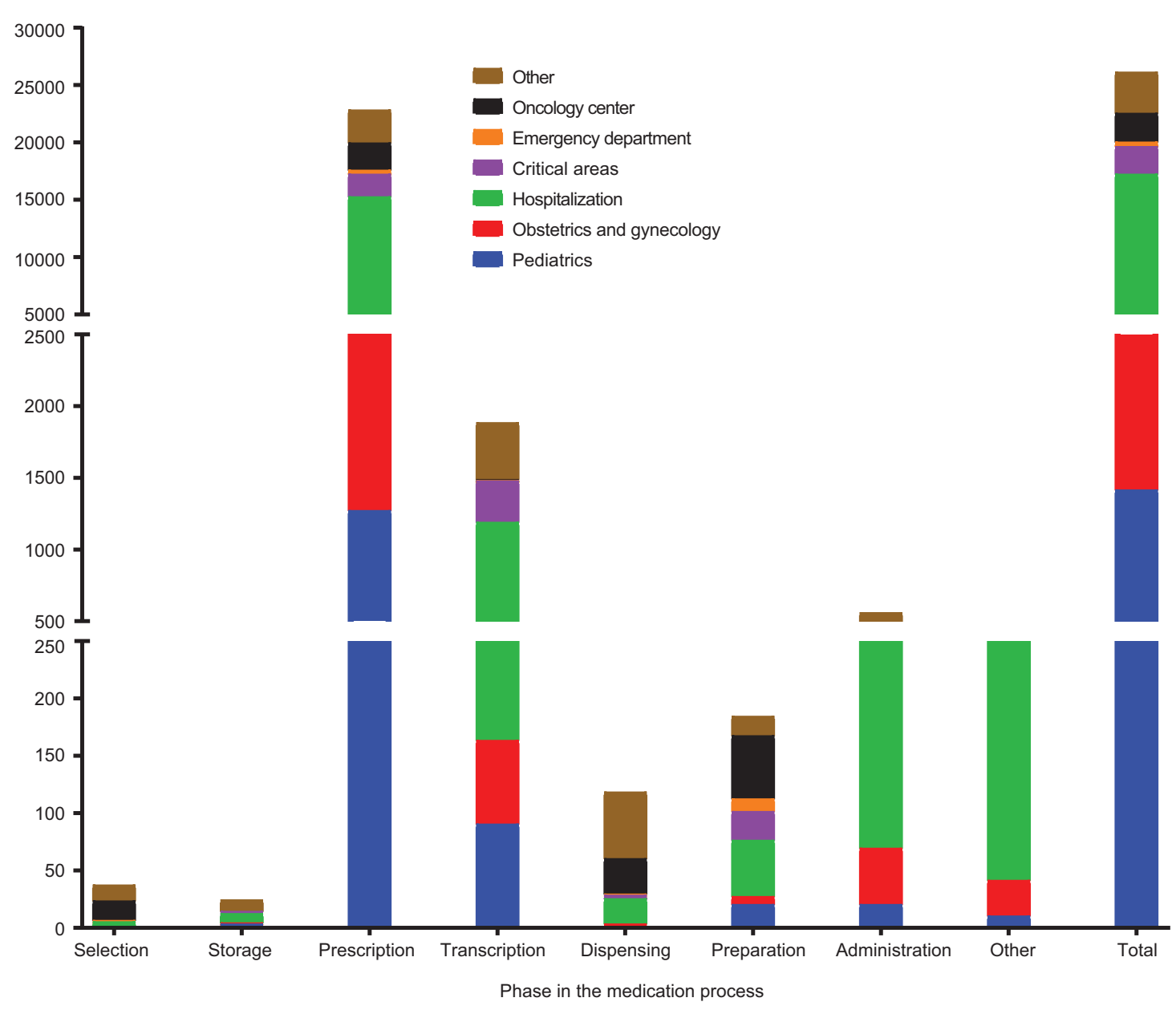

Figure 3. Medication errors per drug prescription and administration phase. Each hospital area is independently represented within the same bar and is directly proportional to the number of medication errors according to the drug prescription and administration phases.

\section{Discussion}

Pharmacovigilance in Mexico has its origin in 1995, when the Ministry of Health implemented it in the drug control and surveillance program with the purpose to strengthen treatment rationalization and optimization, and this way detect potential problems related to medications.

Studies about pharmacovigilance are sparse and have small sample sizes. Therefore, this analysis attempted to obtain the highest possible amount of data, which were collected for more than 3 years, in order to obtain significant results; the sample is the largest thus far reported. Currently, reports generated at Fundación Clínica Médica Sur account for $7 \%$ of those carried out in the country.

It is important for the significance of the joint effort of all medical, nursing and pharmacy personnel under the supervision of the Pharmacovigilance Department to be mentioned. Owing to this collective work, medication errors are found in $8.9 \%$ of total prescriptions, thus placing Fundación Clínica Médica Sur below the $10 \%$ rate reported by other hospital institutions, 5 which reflects on higher safety in patient care.

Treating physicians were responsible for the highest number of medication errors, since they are responsible for most therapeutic decisions. There are different circumstances that might influence on mistakes made by trainee physicians: workload, tiredness and carelessness in medical directions transcription. ${ }^{15,16}$ On the other hand, interns make less errors because they are not allowed to make decisions regarding the patient medication process.

The process that starts with prescription involves numerous intermediate steps until culminating with administration of the drug. The fact that the most common mistake was wrong prescription may be related to deficient information about the drug, lack of 


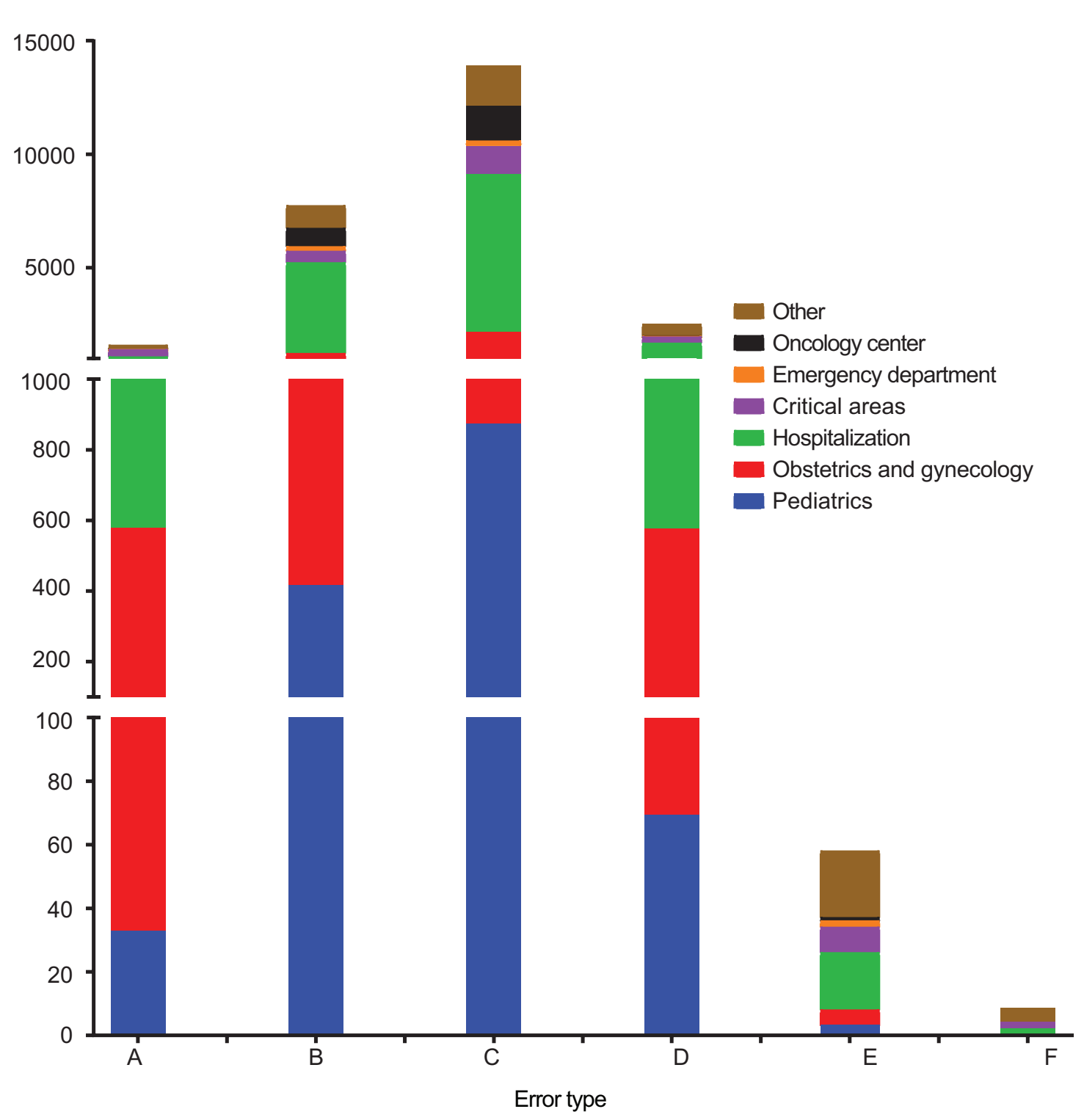

Figure 4. Medication errors per error type (see Table 1). Each hospital area is independently represented within the same bar and is directly proportional to the number of medication errors according to error types.

knowledge about the patient, similarity in drug names or mistakes during verbal prescriptions, among other causes.

The second identified deficiency was during transcription, a phase where situations such as unreadable writing, use of abbreviations, incomplete prescription, incorrect commercial name and health personnel work overload have to be taken into account. ${ }^{16,17}$

It is highly important for medication error consequences to be taken into account. In this study, the most common mistake, in spite of representing an error that affected the patient, did not imply important harm and didn't require additional measures to revert possible harm, which avoided additional expenses. Interestingly, the second most common consequence (type $B$ ) represents an error that didn't affect the patient. Apparently, pharmacovigilance played an important role in the types of error that affected the patient, since none of them caused permanent damage, nor were there associated deaths reported. Among pharmacovigilance departments' duties, promoting correct medical records stands out, and thus reducing collateral adverse reactions, which range from 10 to $12 \%$ in hospitalized patients and are between the fourth and sixth cause of death in developed countries. ${ }^{9}$

For a safe management of drugs, the 5 "rights" have to be put into practice: the right patient, the right drug, the right route, the right dose and the right administration. In surgeries and procedures for them to be safely carried out, the universal protocol has to be followed, 

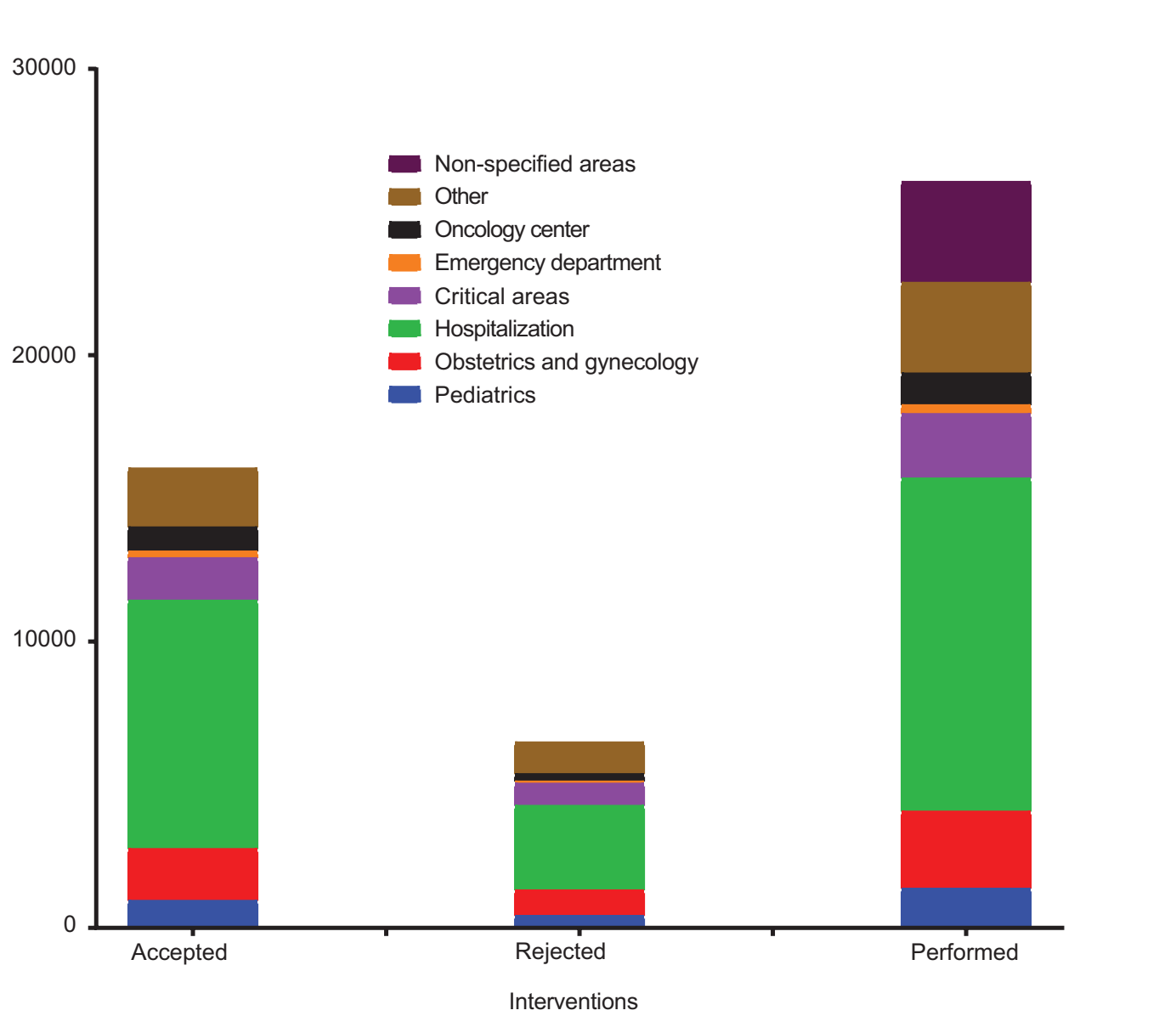

Figure 5. Medication errors per pharmaceutical interventions. Each hospital area is independently represented within the same bar and is directly proportional to the number of medication errors according to the type of pharmaceutical intervention.

which includes the right place, the right procedure and the right patient. The use of diagnostic protocols or guidelines, as well as patient joint responsibility, will help to prevent medication errors by human factors. ${ }^{18}$

Having more information available is useful to assess medication error consequences, its impact on patients in whom it has occurred and the role of pharmacovigilance in the prevention or early solution of such errors and their consequences.

\section{References}

1. Secretaría de Salud. NOM-220-SSA1-2012, instalación y operación de la farmacovigilancia. Diario Oficial de la Federación 2014 Nov 7.

2. Bentsi-Enchill A Couper MR, Duclos P, Edwards IR, Fushimi T, Hartigan-Go K, et al. The importance of pharmacovigilance. United Kingdom: World health organization; 2002.

3. Villegas F. Manual de farmacovigilancia clínica. México: Editorial Panamericana; 2015.

4. Pérez-Peña J. La prescripción médica es un acto científico, ético y legal. Rev Cubana Med Gen Integr. 2002;18(2):114-116.

5. Núñez-Sánchez A, Cornejo-Bravo JM, Pérez-Morales ME. Evaluación de estudios prospectivos sobre errores de medicación en la prescripción: revisión sistemática. Rev Mex Cienc Farm. 2014;45(1):7-14.

6. Quirós-Vega D. Error de medicación: consultas al centro nacional de control de intoxicaciones. Fármacos. 2006;19(1-2):23-30.

7. Lisby M, Nielsen LP, Brock B, Mainz J. How are medication errors defined? A systematic literature review of definitions and characteristics. Int J Qual Health Care. 2010;22(6):507-518.
8. Saucedo-Becerra A, Serrano-Flores FE, Flores-Arcos V, Morales-Olarte E, Santos-García A. Errores frecuentes en la administración de medicamentos intravenosos en pediatría. Rev Enferm Inst Mex Seguro Soc. 2008;16(1):19-26.

9. Pérez-Menéndez-Conde $\mathrm{C}$, Bermejo-Vicedo $\mathrm{T}$, Delgado-Silveira $\mathrm{E}$ Carretero-Accame E. Resultados negativos asociados al uso de medicamentos que motivan ingreso hospitalario. Farm Hosp. 2011;35(5):236-243.

10. De-Bortoli-Cassiani SH, Monzani S, Aparecida A, Trevisani-Fakih F, Perufo-Optiz S, Cardoso T, et al. Identificación y análisis de los errores de medicación en seis hospitales brasileños. Cienc Enferm. 2010;16(1):85-95.

11. Jennane N, Madani N, Oulderrkhis R, Abidi K, Khoudri I, Belayachi J, et al. Incidence of medication errors in a Moroccan medicalintensive care unit. Int Arch Med. 2011;4:32.

12. Rodríguez R, Raymundo $E$, Sánchez JL, Bañales R. Evaluación del sistema de dispensación de medicamentos en el Hospital General de Tijuana. Salud Publica Mex. 2007;49(4):245-246.

13. Nerich V, Limat S, Demarchi M, Borg C, Rohrlich PS, Deconinck E, et al. Computerized physician order entry of injectable antineoplastic drugs: an epidemiologic study of prescribing medication errors. Int J Med Inform. 2010;79(10):699-706.

14. Khalili H, Karimzadeh I, Mirzabeigi P, Dashti-Khavidaki S. Evaluation of clinical pharmacist's interventions in an infectious diseases ward and impact on patient's direct medication cost. Eur J Intern Med. 2013;24(3):227-233.

15. Otero M, Martín R, Robles M, Codina C. Errores de medicación. En: Gamundi MC, eitor. Farmacia hospitalaria. España: FEFG; 2002.

16. Berg GM, Harshbarger JL, Ahlers-Schmidt CR, Lippoldt D. Exposing compassion fatigue and burnout syndrome in a trauma team: a qualitative study. J Trauma Nurs. 2016;23(1):3-10.

17. Carrillo-Esper R, Gómez-Hernández K, De la Torre-León T. Prevalencia de síndrome de Burnout en una unidad de terapia intensiva. Med Sur. 2012;19(4):208-211.

18. Fajardo-Dolci GE, Rodríguez-Suárez J, Gallegos-Alvarado $M$, Córdoba-Ávila MA, Flores-Bravo MC. Percepción de la frecuencia y causas de los eventos adversos por medicación en enfermería. Rev CONAMED. 2009;14(1):22-27. 